\title{
A retrospective database study of oral corticosteroid and bisphosphonate prescribing patterns in England
}

\author{
Christos V. Chalitsios $\mathbb{D}^{1 凶}$, Dominick E. Shaw $\mathbb{D}^{1}$ and Tricia M. McKeever ${ }^{2}$
}

Exposure to oral corticosteroids (OCS) is associated with an increased risk of osteoporosis and fragility fractures. Guidelines suggest bisphosphonate (BP) therapy as the first-line treatment of glucocorticoid-induced osteoporosis (GIOP). This population study used publicly available data, including prescription annual cost analysis and monthly practice-level data. Our aim was to examine the prescribing of OCS and BP at practice level and investigate reasons for variation using a mixed-effect negative binomial regression analysis. There was a rise in OCS and BP prescriptions of 55\% and 1200\% from 1998 to 2018, respectively. Of the 6586 included practices, the median (IQR) of OCS and BP prescriptions were 120.8 (84.8-160.4) and 107.7 (73.8-147.4) per 1000 patients, respectively. Asthma and chronic obstructive pulmonary disease (COPD) were significantly associated with OCS use $(p<0.0001)$, but only COPD was associated with BP use $(p<0.0001)$. Higher OCS prescribing rates were associated with higher BP prescribing rates (5th to 1 st quintile-IRR $=1.99 ; 95 \% \mathrm{Cl}: 1.88-2.10$ ). Practice list size, deprivation and advanced age were all associated with both drugs $(p<0.0001)$. In conclusion, although OCS use is positively associated with BP prescription, variation among practices and CCGs exists. The variation in prescribing suggests there is still a need to improve GIOP prevention.

npj Primary Care Respiratory Medicine (2020)30:5 ; https://doi.org/10.1038/s41533-020-0162-6

\section{INTRODUCTION}

Oral corticosteroids (OCS) (glucocorticoids) are used to treat chronic conditions including autoimmune, ${ }^{1}$ and respiratory diseases. ${ }^{2,3}$ Asthma and COPD are two of the most common indications for prolonged OCS use (more than 90 days). ${ }^{4}$ Both short-term (5-90 days) and prolonged exposure to OCS can lead to deleterious effects ${ }^{5,6}$ including bone loss resulting in osteoporosis and fragility fracture. ${ }^{7}$ Bone loss is substantial and rapid during the first months of the OCS treatment. ${ }^{8}$ Patients with severe asthma exposed to prednisolone $5 \mathrm{mg}$ per day are more likely to be diagnosed with osteoporosis $(\mathrm{OR}=6.5)$ and have a fracture $(O R=1.5)$ compared to those without asthma. ${ }^{9}$ After OCS initiation, spine fracture risk increases by $55 \%$ with exposure at doses as low as prednisone $2.5 \mathrm{mg}$ per day, whereas hip fracture risk goes up by $50 \%$ among patients exposed to $2.5-7.5 \mathrm{mg}$ per day. ${ }^{10,11}$

Fragility fractures are also associated with substantially increased healthcare costs, morbidity, and mortality, ${ }^{12,13}$ and guidelines suggest all patients exposed to any dose of OCS for more than 3 months should be considered for BP therapy to prevent glucocorticoid-induced osteoporosis (GIOP). ${ }^{14,15}$ The bisphosphonate class is effective in reducing bone loss and fragility fracture risk. ${ }^{16,17}$ Despite this, only a minority of patients with increased fragility fracture risk receive appropriate therapy. ${ }^{18,19}$ There are no specific guidelines for GIOP in asthma or COPD and the size of the potential problem is not well established.

We are unaware of any published U.K. research investigating the trends in OCS and anti-GIOP therapy (BP) prescribing. Our aim was to comprehensively assess OCS and BP prescribing patterns, at practice level, using primary care data from England and to investigate factors associated with their prescribing, in order to gain a better understanding of prescribing enabling us to reduce prescribing variation and optimise GIOP prevention.

\section{RESULTS}

Practice characteristics

In our analysis, we included 195 Clinical Commissioning Groups (CCGs) containing 6586 practices after the exclusion of 507. In 2018, the median (IQR) OCS and BP prescriptions per 1000 patients was 120.8 (84.8-160.4) and 107.7 (73.8-147.4), respectively. The characteristics of practices are summarised in Table 1.

Long-term patterns and ratio between OCS and BP prescriptions Prednisolone was the most frequently prescribed OCS. There was a steady increase in OCS prescriptions over time (Fig. 1a). In 1998, there were 95 OCS prescriptions per 1000 population increasing to 140 in 2018 (55\% rise). The cost of OCS was $£ 250$ per 1000 population until 2006, with a noticeable increase up to just less than $£ 2000$ the following years (Supplementary Fig. 1).

There was an increase in bisphosphonate prescribing rates over time (Fig. 1b). In 1998, there were 10 BP prescriptions per 1000 population, while the total prescriptions reached 120 in 2018 (1200\% increase). The most prescribed bisphosphonate was alendronic acid. BP cost peaked at $£ 3200$ per 1000 population in 2005; however, there was a reduction to $£ 101$ by 2018 (Supplementary Fig. 1).

There were 0.99 OCS prescriptions per 1 BP item in 2015; however, this relationship changed slightly to 1.16 by 2018 (Table 2).

Variations among practices and CCGs for OCS and BP items In 2018, there was a significant variation between OCS ( $m=129.6$, $\mathrm{SD}=38.9)$ and $\mathrm{BP}(m=118.5, \mathrm{SD}=34.2)$ prescription per 1000 patients; $t=6.27, p<0.0001$. OCS prescription varied between 48 and 239 and BP ranged from 38 to 207 prescriptions per 1000 patients across CCGs. Sixty out of 195 CCGs prescribed less OCS

\footnotetext{
${ }^{1}$ NIHR Division of Respiratory Medicine, University of Nottingham, Nottingham, UK. ${ }^{2}$ Division of Epidemiology and Public Health, University of Nottingham, Nottingham, UK.

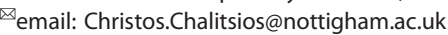


than BP items, and 135 more OCS than BP items per 1000 patients (Fig. 2).

Factors associated with OCS and BP prescribing

We found that OCS prescriptions were associated with the factors listed in Table 3 apart from the QOF score and percentage of

Table 1. Characteristics of practices included in the analysis (from January 2018 to December 2018).

\begin{tabular}{llll}
\hline & Median & IQR \\
\hline Asthma prevalence (\%) & 6.0 & $5.1-6.8$ \\
COPD prevalence (\%) & 1.8 & $1.3-2.5$ \\
Practice list size & 7478 & $4664-11,270$ \\
Patients with long-term health conditions (\%) & 51.7 & $45.5-59.1$ \\
Patients over 65 years old (\%) & 17.4 & $12.2-21.8$ \\
Quality Outcomes Framework score & 549.0 & $534.7-556.8$ \\
OCS prescribed items per 1000 patients & 120.8 & $84.8-160.4$ \\
BP prescribed items per 1000 patients & 107.7 & $73.8-147.4$ \\
\hline COPD chronic obstructive pulmonary disease, OCS oral corticosteroids, BP \\
bisphosphonates.
\end{tabular}

patients with a long-term health disease. Asthma and COPD were significantly associated with the OCS use $(p<0.0001)$. The percentage of patients aged 65 years old or more was the strongest predictor of OCS prescriptions $(p<0.0001)$. Practices in the highest quintile prescribed 1.74 times more OCS (IRR $=1.74$; 95\% Cl: 1.64-1.84) than those in the lowest one. Practise list size was also a positive predictor of OCS prescribing $(p<0.0001)$ and the most deprived areas were less likely to prescribe less OCS than the least deprived areas (IRR $=0.84 ; 95 \% \mathrm{Cl}$ : 0.80-0.88).

OCS were associated with BP prescriptions $(p<0.0001)$, with higher OCS prescribing rates to have been associated with higher $\mathrm{BP}$ prescribing rates (5th to 1 st quintile-IRR $=1.99 ; 95 \% \mathrm{Cl}$ :

Table 2. Trends in ratio between oral corticosteroids and bisphosphonates over the period of 2015-2018.

\begin{tabular}{llll}
\hline Year & OCS items & BP items & Ratio scale (OCS/BP) \\
\hline 2015 & $7,781,584$ & $7,836,568$ & 0.99 \\
2016 & $7,958,014$ & $7,479,733$ & 1.06 \\
2017 & $7,911,005$ & $7,062,931$ & 1.12 \\
2018 & $7,799,798$ & $6,728,997$ & 1.16 \\
\hline
\end{tabular}

OCS oral corticosteroids, BP bisphosphonates.

(a) Total items for all OCS

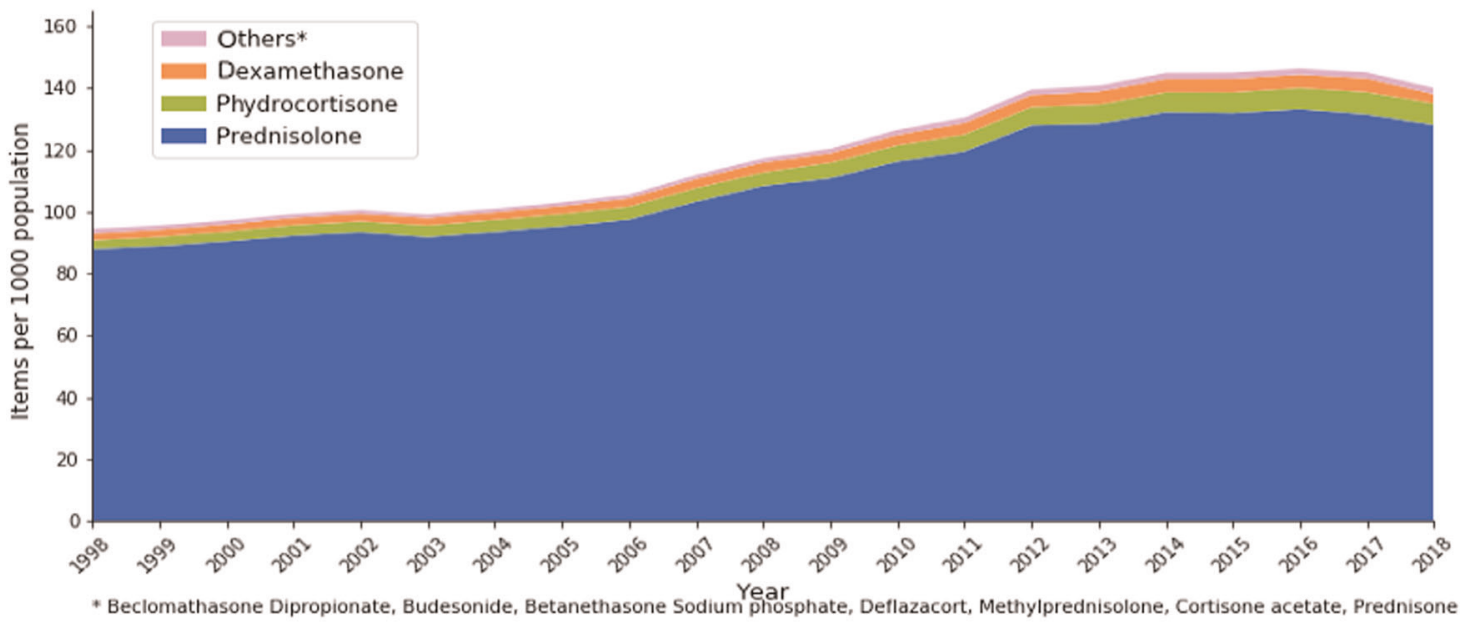

(b) Total items for all bisphosphonates

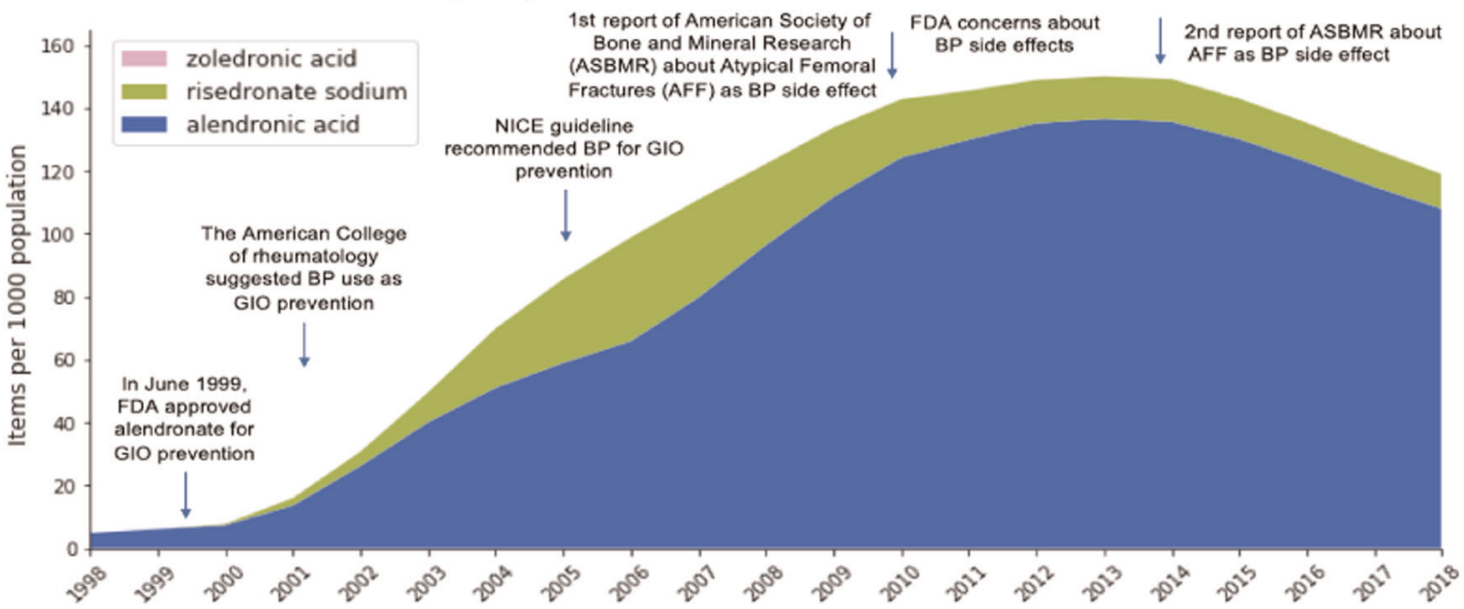

Fig. 1 Long-term prescribing patterns. Total (a) oral corticosteroids (OCS) and (b) bisphosphonates (BP) prescribed items per 1000 population over the period from 1998 to 2018. The arrows provide factors that may have affected their prescribing. 
a) OCS prescriptions per 1,000 patients

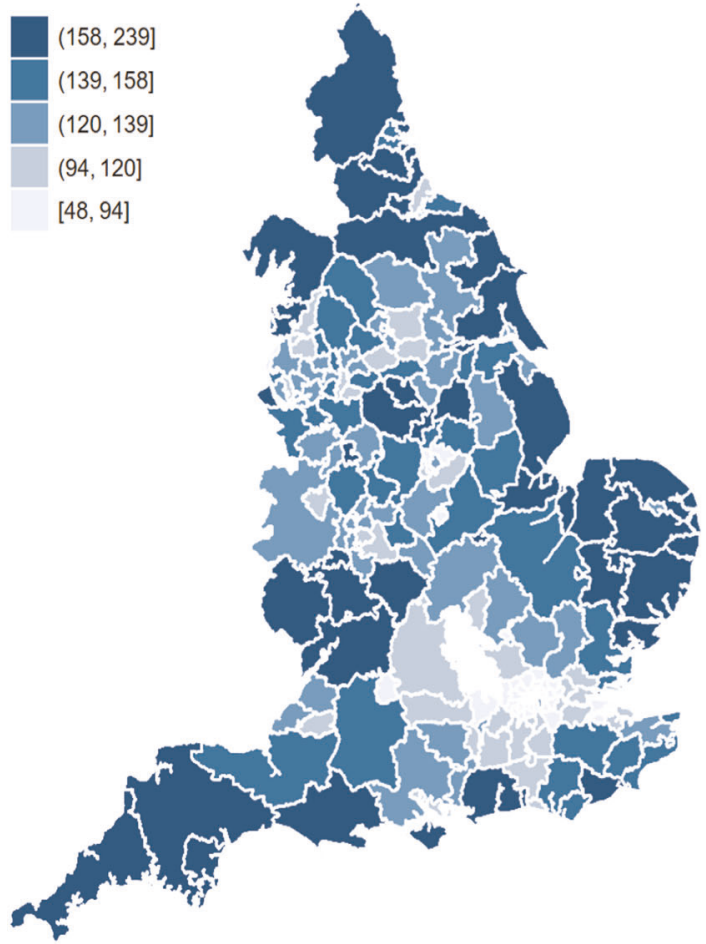

b) BP prescriptions per 1,000 patients

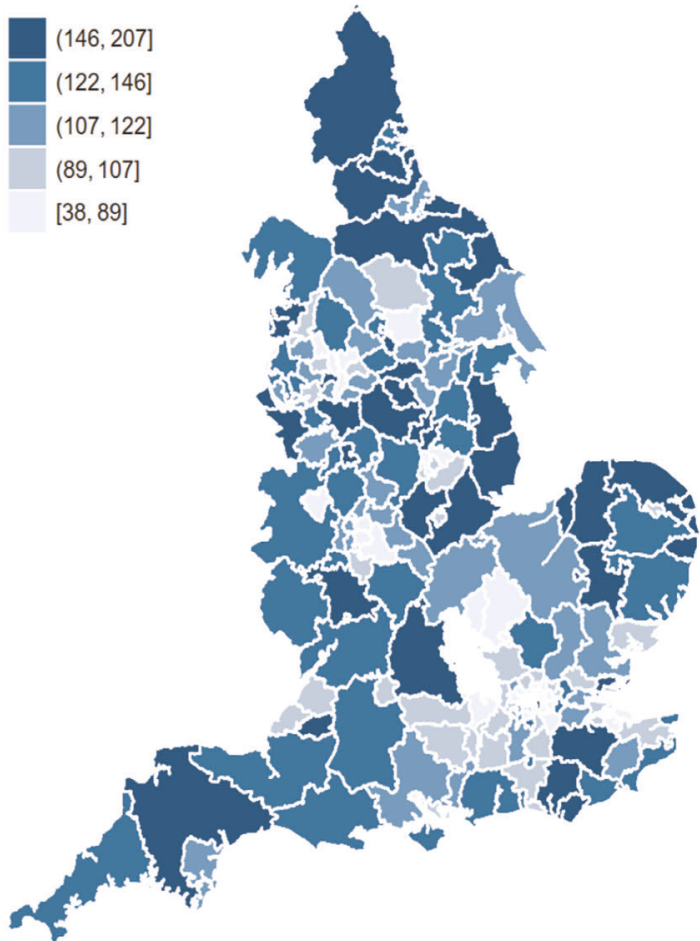

Fig. 2 Geographical variation in prescribing. Geographical variations in (a) oral corticosteroids (OCS) and (b) bisphosphonates (BP) prescribed items categorised into quantiles among Clinical Commissioning Groups from January to December 2018.

1.88-2.10) (Table 4). COPD was significantly associated with a BP prescription $(p<0.0001)$, but asthma was not $(p=0.6848)$. Practices located in more deprived areas were $28 \%$ less likely to prescribe less BP than the least deprived practices (IRR $=0.72 ; 95 \%$ $\mathrm{Cl}$ : 0.68-0.77).

The CCG to which a practice belongs was significantly associated with prescribing rates and accounted for $11 \%$ and $5 \%$ of the variation in OCS and BP prescribing, respectively.

We also found similar results in the other years (Supplementary Tables 4-12).

\section{DISCUSSION}

Overall, we observed an increase in prescribed OCS and BP items between 1998 and 2018. We found large variation in prescribing rates across practices in England. Asthma and COPD were significantly related with OCS prescriptions, but only COPD with BP. Patient list size, deprivation and advanced age were all associated with both the drugs. The CCG to which each practice belongs also contributed to the prescribing variation. Finally, OCS was positively associated with BP prescriptions.

The increase in BP prescriptions (2000-2010) could be explained by the uptake of clinical guidance. The FDA approved alendronate use in June $1999^{20}$; the first guidance for the GIOP prevention from the American College of Rheumatology was published in $2001^{21}$ and NICE recommended BP as first-line GIOP prophylaxis in $2005 .^{22}$ The plateauing of BP prescriptions from 2010 with a downward trend from 2015, in contrast with the steady OCS prescriptions, may reflect the FDA concerns ${ }^{23,24}$ about BP side effects such as osteonecrosis of the jaw and atypical femoral fractures. In 2014, the release of the second report of American Society of Bone and Mineral Research provided a more robust evidence about the atypical fractures as BP side effects probably was the reason for a further decrease. ${ }^{25}$ An investigation in the USA found a similar 50\% reduction in BP use between 2008 and 2012 following concerns about their safety. ${ }^{26}$ However, these side effects might be greater concern in younger age groups, as they will benefit less using BP. Our findings are also consistent with two other studies that found a steady increase in BP use from 2000 onwards. $^{27,28}$

Establishing an optimal ratio of BP to OCS prescription is challenging. The management of multi-morbidities makes prescribing decisions complex and although prescriptions should be guideline informed, they should not be guideline directed. ${ }^{29,30}$ Initiation of BP medication may also depend on how long past 3 months OCS exposure is expected. There is good evidence that the benefits of BP in preventing GIOP outweigh the risks ${ }^{25,31,32}$ and bone mineral density testing is recommended within 6 months after OCS initiation, repeating it every 1-3 years. ${ }^{33}$ Other potential options for GIOP may be recommended (Vitamin $D, H R T)$, but have less data.

Our analysis demonstrates geographical variation across practices and CCGs in prescribing rates of both classes of drugs and in between the drugs. Apart from variation associated with practice factors, our results revealed that CCGs accounted for variation in each medication that may indicate differences in prescribing policy. Interestingly, prescribing differences related to deprivation, in terms of OCS, may reflect inequity of access to treatment difference, whereas in BP rates, may reflect access to DEXA scanning. Our findings are consistent with another UK study that found marked regional differences in the anti-osteoporosis prescribing rates. ${ }^{26}$ Other studies have also confirmed the impact of deprivation and the other examined factors on variation in drug prescribing. $^{34-36}$

Despite the proven benefits of BP as an osteoporosis therapy, there is evidence that they are underutilised both in the UK and 
Table 3. Oral corticosteroids prescribing rates in 2018 , stratified by seven practice characteristics and two respiratory diseases in a negative binomial model reporting incidence rate ratio.

\begin{tabular}{|c|c|c|c|c|c|}
\hline & Quintile range & $\begin{array}{l}\text { Median OCS prescription } \\
\text { per } 1000 \text { patients }\end{array}$ & $\begin{array}{l}\text { Univariate model } \\
\text { IRR }(95 \% \mathrm{CI})^{\mathrm{a}}\end{array}$ & $\begin{array}{l}\text { Multivariate model } \\
\text { IRR }(95 \% \mathrm{CI})^{\mathrm{b}}\end{array}$ & $p$ value $^{c}$ \\
\hline \multirow[t]{3}{*}{ Asthma prevalence (\%) } & $\leq 4.85$ & 69.26 & Reference & Reference & \multirow[t]{3}{*}{$<0.0001$} \\
\hline & $5.68-6.29$ & 128.56 & $1.69(1.61-1.76)$ & $1.18(1.13-1.23)$ & \\
\hline & $6.30-6.98$ & 144.31 & $1.90(1.82-1.98)$ & $1.23(1.18-1.29)$ & \\
\hline \multirow{4}{*}{ COPD prevalence (\%) } & $1.18-1.65$ & 107.95 & $1.48(1.42-1.55)$ & $1.13(1.09-1.17)$ & \multirow{4}{*}{$<0.0001$} \\
\hline & $1.66-2.10$ & 128.56 & $1.77(1.70-1.85)$ & $1.20(1.15-1.25)$ & \\
\hline & $2.11-2.69$ & 144.67 & $1.95(1.87-2.04)$ & $1.26(1.21-1.33)$ & \\
\hline & $2.70-10.45$ & 155.17 & $2.10(2.01-2.20)$ & $1.34(1.27-1.41)$ & \\
\hline Practice list size & $\leq 4,127$ & 112.14 & Reference & Reference & $<0.0001$ \\
\hline \multirow[t]{5}{*}{ QOF score } & $\leq 529.81$ & 111.24 & Reference & Reference & \multirow[t]{5}{*}{0.2579} \\
\hline & $529.82-545.04$ & 115.02 & $1.05(1.01-1.11)$ & $1.03(0.99-1.07)$ & \\
\hline & $545.05-552.16$ & 118.01 & $1.08(1.03-1.13)$ & $1.02(0.99-1.06)$ & \\
\hline & $552.17-557.84$ & 128.73 & $1.15(1.10-1.21)$ & $1.01(0.98-1.06)$ & \\
\hline & $557.85-559$ & 137.49 & $1.24(1.18-1.30)$ & $1.02(0.98-1.07)$ & \\
\hline \multirow[t]{5}{*}{$\%$ over 65 years old } & $\leq 11.00$ & 68.16 & Reference & Reference & \multirow[t]{5}{*}{$<0.0001$} \\
\hline & $11.01-15.63$ & 106.01 & $1.50(1.44-1.56)$ & $1.24(1.20-1.28)$ & \\
\hline & $15.64-18.86$ & 126.68 & $1.78(1.70-1.85)$ & $1.39(1.34-1.46)$ & \\
\hline & $18.87-22.77$ & 139.56 & $1.95(1.87-2.04)$ & $1.49(1.42-1.57)$ & \\
\hline & $\geq 22.78$ & 172.23 & $2.40(2.30-2.50)$ & $1.74(1.64-1.84)$ & \\
\hline \multirow{3}{*}{ IMD score } & - & 122.00 & $0.96(0.91-1.01)$ & $0.87(0.83-0.90)$ & \multirow{3}{*}{$<0.0001$} \\
\hline & - & 115.85 & $0.89(0.85-0.94)$ & $0.85(0.81-0.88)$ & \\
\hline & Most deprived & 110.01 & $0.88(0.84-0.92)$ & $0.84(0.80-0.88)$ & \\
\hline
\end{tabular}

USA. ${ }^{19,28}$ Addressing this issue will hinge on education in both primary and secondary care, and provision of suitable guidelines. One practical solution in healthcare systems that use electronic records/prescribing would be to flag patients who meet BP criteria based on age, gender and OCS use. Alerts already occur for a number of conditions (including excess salbutamol use) and this flag could be incorporated into the chronic disease review.

Although there is clear guidance on OCS and BP therapy, there is no current recommendation for BP therapy for inhaled corticosteroid (ICS) users, despite evidence supporting an increased osteoporotic fracture risk related to ICS. ${ }^{37-39}$ It is best practice to review ICS dose and use the lowest dose possible to maintain asthma control. ${ }^{40}$

We also found that the rise in OCS cost was mainly driven by a steep increase in price of hydrocortisone tablets from 2008 onwards; and BP prescribing went up as BP cost went down after 2005.

To our knowledge, this is the first study to examine the OCS and BP prescribing patterns and their association with practice-level factors. We use real prospectively collected prescribing data based on NHS Digital files and included practices and CCGs covering the entire country. There are some limitations; we could not evaluate prescriptions in secondary care. Secondly, we were not able to 


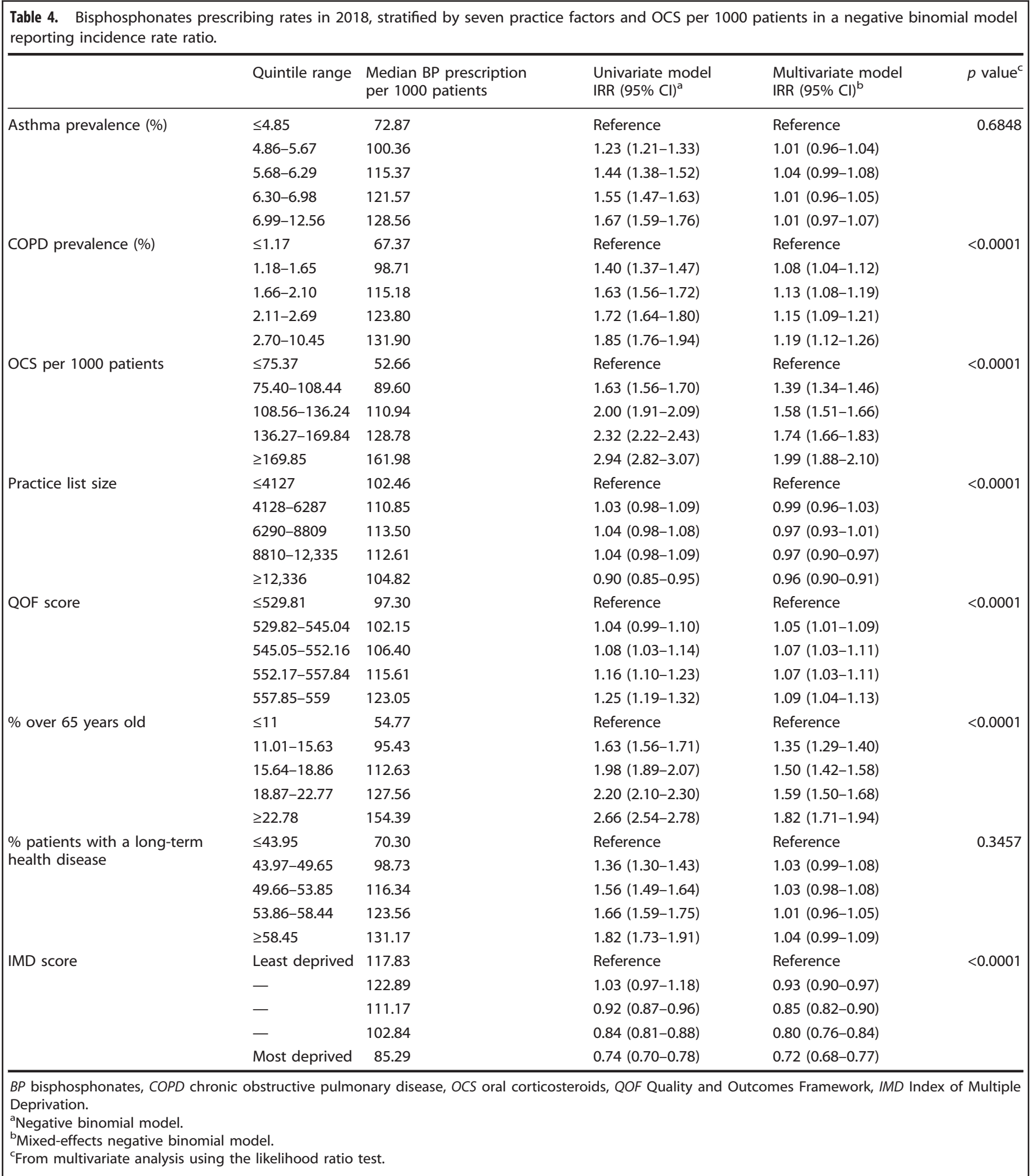

know the indication for each prescription. Thirdly, we could not perform individual-level analysis in order to identify the OCS prescriptions that need BP therapy according to the guidelines' recommendations.

In conclusion, the overall levels of OCS and BP prescription have increased since 1998. Concerns about BP adverse effects may account for a latter reduction in BP prescriptions in contrast to steady or increased OCS prescriptions. We found clear variation in OCS and $\mathrm{BP}$, and this unwarranted variation appears to be driven to a large extent by factors including deprivation, patient list size and CCGs. The variation in prescribing suggests there is still a need to improve GIOP prevention. 


\section{METHODS}

\section{Data sources}

We used national data based on the annual prescription cost analysis (PCA) from 1998 to 2018, and monthly practice-level data files by NHS Digital from January 2015 to December 2018.

The annual prescription cost analysis data files provide details of the number of items and net ingredient cost of all prescriptions for each medication dispensed in the community in England. Data were normalised by converting to relative figures per 1000 population using mid-year population estimate for England. ${ }^{41}$ The monthly data published by NHS Digital consist of one row for each medication providing information about the prescribing volume and cost for each practice in England. These data are based on claims from community pharmacies and contains items that have been dispensed.

We extracted the data from OpenPrescribing (https://openprescribing. net/) database. OpenPrescribing is built by EBM Data Lab and provides a search interface onto the raw prescribing data files published by NHS, making the access to this complex information easier in order for these data to be more impactful in real world. There are approximately 900 million rows of these data. New data are released every month; however, they are 2 months behind (i.e. March's prescribing data are published in May). OpenPrescribing has data from the past 5 years without containing any patient information and indication of the treatment length.

\section{Study design}

We conducted a retrospective cohort with ecological elements study on all English practices and CCGs, measuring patterns in OCS and BP prescribing items over time at practice level. We described the ratio in prescribing rate between OCS and BP and measured any variation among CCGs geographically. We also matched the monthly practice-level data with open access data derived from Public Health England to investigate for reason for any variation in OCS and BP prescribing at practice and CCG level.

\section{Drugs extraction}

We used prescribed "items" as a measure of prescribing. A prescription item refers to a single supply of a medicine prescribed on a prescription form. If a prescription form includes three medicines, it is counted as three prescription items.

We extracted prescribing and cost data for the following OCS as are stated on OpenPrescribing.net: Beclometasone Dipropionate (Systemic) (Brand name: Clipper), Budesonide (Brand names: Entocort, Budenofalk) derived from section "1.2.5: Corticosteroids" of the British National Formulary (BNF) book. Betamethasone Sodium Phosphate (brand names: Betnesol, Betameth sod phos, all systemic), Cortisone Acetate (brand name: Cortisone acet), Deflazacort (brand name: Calcort), Dexamethasone (brand name: Dexameth (systemic)), Hydrocortisone (brand name: Hydrocortone, Hydrocort (systemic)), Methylprednisolone (brand name: Medrone (systemic)), Prednisolone (brand name: Prednisolone (systemic)), and Prednisone (brand name: Lodotra) were derived from section "6.3.2: Glucocorticoid therapy" of the BNF (Supplementary Table 2).

We extracted data about bisphosphonates following guidance from the National Osteoporosis Guideline Group. ${ }^{12}$ These were: Alendronic acid (brand names: Alendronic acid, Fosamax), risedronate sodium (brand names: Actonel, Risedronate sod) and Zoledronic acid (brand name: Zometa). All BP were checked against section "6.6.2: Bisphosphonates and other drugs" of the BNF (Supplementary Table 1).

\section{Long-term patterns}

Data from OpenPrescribing.net based on PCA were obtained describing the annual patterns in OCS and BP prescribing items and cost per 1000 population from 1998 to 2018 . We aggregated the total annual items/cost per 1000 population of each OCS/BP type. We created stacked graphs to depict the annual volume of each chemical of each drug per 1000 population.

\section{Ratio between OCS and BP prescriptions}

We extracted monthly data about OCS and BP prescribed items per CCG from January 2015 to December 2018. We aggregated the monthly items of each CCG per year obtaining the total annual number of both OCS and $\mathrm{BP}$ items. The ratio between the above classes of drugs was calculated using:

Ratio $=\left(\frac{\text { Total OCS items the year of interest }}{\text { Total BP items the year of interest }}\right)$.

Variations among CCGs for OCS and BP items

In 2012, each practice was grouped into CCGs. The CCGs are responsible for the planning and commissioning of healthcare in a local community. To examine geographical variations in OCS and BP prescribing in 2018, practices were grouped by CCG. The prescribing rate per 1000 patients of dispensed OCS/BP for each CCG was derived by dividing the total number of prescribing items by the mean patient list size over the year in each CCG, multiplied by 1000 . Then, they were categorised into quantiles.

\section{Associations between OCS and BP prescribing}

After calculating the rate of OCS and BP items per 1000 patients per practice, we determined other independent variables to examine which indicators were associated with OCS and BP prescription in 2018. We repeated the analysis for 2015, 2016 and 2017.

Data were extracted on the following factors from the Public Health England (https://fingertips.phe.org.uk/profile/general-practice/data): the percentage of (a) asthma diagnosis, (b) COPD diagnosis, (c) patients over 65 years old, (d) patients with a long-term health condition defined as the percentage of people who answered "Yes" in the practices' patient survey (http://www.gp-patient.co.uk/practices-search) question: "Do you have any long-term physical or mental health conditions, disabilities or illnesses?", (e) the Index of Multiple Deprivation (IMD) score, and (f) the mean practice list size.

All of the above figures were derived from the correspondence year of interest apart from the IMD score which was available only for 2015; we used this score for the analysis in each year of interest. All of the above indicators were obtainable based on the CCGs which were active in 2017/ 2018. Furthermore, we extracted data about the Quality and Outcomes Framework Score (QOF) score (https://digital.nhs.uk/data-and-information/ publications/statistical/quality-and-outcomes-framework-achievementprevalence-and-exceptions-data) by practice. We used the 2017/2018 QOF score not only in the analysis for the year 2017 but also in 2018 as the 2018/2019 QOF score had not been released yet. Additionally, we excluded practices without a QOF or having a list size less than 1000 patients (Supplementary information, Practice exclusion).

\section{Statistical methods}

Practices' characteristics as well as OCS and BP prescriptions were analysed using descriptive analysis reporting them as median along with IQR. A paired $t$ test was performed examining the significance of variation between OCS and BP prescription among CCGs. To examine the association between OCS/BP per 1000 patients and the factors, we conducted a negative binomial regression analysis. We stratified the rate of OCS and BP prescribing for each one of the investigating factors. We put these factors in the model. We also split the OCS per 1000 patients into quintiles and put them in the BP analysis. Afterwards, as healthcare policies and commissioning differ among CCGs, we used a mixed-effect negative binomial model, defining the rate of OCS and BP prescriptions per 1000 patients as the dependent variable. We used the factors defined above as fixed-effects explanatory variables, and the CCG of each practice as a random-effect variable. The variables were grouped into quintiles to allow for nonlinearity of effects. IRRs with a $95 \% \mathrm{Cl}$ were used to determine the strength of associations and $R^{2}$ to show the value and significance of variance associated with CCG grouping. $P$ values $<0.05$ were considered statistically significant. Practices with missing values were less than $0.25 \%$.

Python was used for data management. Both graphs and maps were constructed with Python. Statistical analysis was conducted by using Stata, version 16.

\section{Reporting summary}

Further information on experimental design is available in the Nature Research Reporting Summary linked to this article. 


\section{DATA AVAILABILITY}

The datasets generated and/or analysed during the current study are available from the corresponding author on reasonable request. The datasets can be found on https://doi.org/10.6084/m9.figshare.11590707.

\section{CODE AVAILABILITY}

The program codes used during the current study are available from the corresponding author on reasonable request.

Received: 8 July 2019; Accepted: 16 January 2020; Published online: 13 February 2020

\section{REFERENCES}

1. National Institute for Health and Care Excellence. Rheumatoid arthritis in adults: management. https://www.nice.org.uk/guidance/ng100 (2018).

2. Global Initiative for Asthma. Global strategy for asthma management and prevention. https://ginasthma.org/download/832/ (2018).

3. Global Initiative for Chronic Obstructive Lung Disease. Global strategy for the diagnosis, management, and prevention of chronic obstructive pulmonary disease. https://goldcopd.org/ (2019).

4. Fardet, L. \& Petersen, I. Monitoring of patients on long-term glucocorticoid herapy: a population-based cohort study. Medicine 94, 1-10 (2015).

5. Waljee, A. K. et al. Short term use of oral corticosteroids and related harms among adults in the United States: population based cohort study. BMJ 357, 1-8 (2017)

6. Rice, J. B., White, A. G., Scarpati, L. M., Wan, G. \& Nelson, W. W. Long-term systemic corticosteroid exposure: a systematic literature review. Clin. Ther. 39, 2216-2229 (2017).

7. Weinstein, R. S. Glucocorticoid-induced bone disease. N. Engl. J. Med. 365, 62-70 (2011).

8. LoCascio, V. et al. Bone loss in response to long-term glucocorticoid therapy. Bone Min. 8, 39-5 (1990).

9. Sweeney, J. et al. Comorbidity in severe asthma requiring systemic corticosteroid therapy: cross-sectional data from the Optimum Patient Care Research Database and the British Thoracic Difficult Asthma Registry. Thorax 71, 39-46 (2016).

10. van Staa, T. P., Leufkens, H. G., Abenhaim, L., Zhang, B. \& Cooper, C. Oral corticosteroids and fracture risk: relationship to daily and cumulative doses. Reumatology 69, 1521-1537 (2017).

11. van Staa, T. P., Leufkens, H. G., Abenhaim, L., Zhang, B. \& Cooper, C. Use of oral corticosteroids and risk of fractures. J. Bone Miner. Res. 15, 993-1000 (2000).

12. Svedbom, A. et al. Osteoporosis in the European Union: a compendium of country-specific reports. Arch. Osteoporos. 8, 1-218 (2013).

13. Johnell, O. et al. Mortality after osteoporotic fractures. Osteoporos. Int. 15, 15-38 (2004).

14. Hoes, J. N. et al. European League Againist Reumatism evidence-based recommendations on the management of systemic glucocorticoid therapy in rheumatic diseases. Ann. Rheum. Dis. 66, 1560-1567 (2007).

15. National Osteoporosis Guideline Group. NOGG 2017: Clinical guideline for the prevention and treatment of osteoporosis. https://www.sheffield.ac.uk/NOGG/ NOGG\%20Guideline\%202017.pdf (2018).

16. Murad, H. M. et al. Comparative effectiveness of drug treatments to prevent fragility fractures: a systematic review and netwotk meta-analysis. J. Clin. Endocrinol. Metab. 97, 1871-1880 (2012).

17. MacLean, C. et al. Systematic review: comparative effectiveness of treatments to prevent fractures in men and women with low bone density or osteoporosis. Ann. Intern. Med. 148, 197-213 (2008).

18. Albaum, J. M., Youn, S., Levesque, L. E., Gershon, A. S. \& Cadarette, S. M. Osteoporosis management among chronic glucocorticoid users: a systematic review. J. Popul Ther. Clin. Pharm. 21, e486-e504 (2014).

19. Giangregorio, L. et al. Fragility fractures and the osteoporosis care gap: an international phenomenon. Semin. Arthritis Rheum. 35, 293-305 (2006).

20. Merck Research Laboratories. Approval letter. https://www.accessdata.fda.gov/ drugsatfda_docs/nda/99/020560S13_Fosamax_appltr.pdf (1999)

21. Americal College off Rheunatology Ad Hoc Committee on GlucocorticoidInduced Osteoporosis. Recommendations for the prevention and treatment of glucocorticoid- induced osteoporosis. Arthritis Rheum. 44, 496-503 (2001).

22. National Institute for Clinical Excellence. Bisphosphonates (Alendronate, Etidronate, Risedronate), Selective Oestrogen Receptor Modulators (Raloxifene) and Parathyroid
Hormone (Teriparatide) for the Secondary Prevention of Osteoporotic Fragility Fractures in Postmenopausal Women (NICE, London, 2005).

23. U.S. Food and Drug Administration. FDA drug safety communication: safety update for osteoporosis drugs, bisphosphonates, and atypical fractures. https:// www.fda.gov/Drugs/DrugSafety/ucm229009.htm (2010).

24. Balkhi, B., Seoane-Vazquez, E. \& Rodriguez-Monguio, R. Changes in the utilization of osteoporosis drugs after the 2010 FDA bisaphosphonate drug safety communication. Saudi Pharm. J. 26, 238-243 (2018).

25. Shane, E. et al. Atypical subtrochanteric and diaphyseal femoral fractures: second report of a task force of the American Society for Bone and Mineral Research. J. Bone Min. Res 29, 1-23 (2014).

26. Jha, S., Wang, Z., Laucis, N. \& Bhattacharyya, T. Trends in media reports, ora bisphosphonate prescriptions, and hip fractures 1996-2012: an ecological analysis. J. Bone Min. Res. 30, 2179-2187 (2015).

27. Wang, L., Shawn, T. C., Moineddin, R. \& Upshur, R. E. G. Osteoporosis prescribing trends in primary care: a population-based retrospective cohort study. Prim. Health Care Res. Dev. 14, 1-6 (2013).

28. van der Velde, R. Y. et al. Trends in oral anti-osteoporosis drug prescription in the United Kingdom between 1990 and 2012: Variation by age, sex, geographic location and ethnicity. Bone 94, 50-55 (2017).

29. Barnett, K. et al. The epidemiology of multimorbidity in a large cross-sectional dataset: implications for health care, research and medical education. Lancet $\mathbf{3 8 0}$, 37-43 (2012).

30. Wallace, E. et al. Managing patients with multimorbidity in primary care. $B M J \mathbf{2 0}$ 350:h176 (2015).

31. Kennel, K. A. \& Drake, M. T. Adverse Effects of Bisphosphonates: Implications for Osteoporosis Management. Mayo Clin. Proc. 84, 632-638 (2009).

32. Khosla, S. et al. Benefits and risks of bisphosphonate therapy for osteoporosis. J. Clin. Endocrinol. Metab. 97, 2272-2282 (2012).

33. Buckley, L. et al. 2017 Americal College of Reumatology guideline for the prevention and treatment of glucocorticoid-induced osteoporosis. Arthritis Reumatol. 69, 1521-1537 (2017).

34. Curtis, H. J. et al. Opioid prescribing trends and geographical variation in England 1998-2018: a retrospective database study. Lancet Psychiatry 6, 140-150 (2019).

35. Ashworth, M., Lloyd, D., Smith, R. S., Wagner, A. \& Rowlands, G. Social deprivation and statin prescribing: a cross-sectional analysis using data from the new UK general practitioner "Quality and Outcomes Framework". J. Public Health 29, 40-47 (2006).

36. Ettinger, B., Chidambaran, P. \& Pressman, A. Prevalence and determinants of osteoporosis drug prescription among patients with high exposure to glucocorticoid drugs. Am. J. Manag Care 7, 597-605 (2001).

37. van Staa, T. P., Leufkens, H. G. \& Cooper, C. Use of inhaled corticosteroids and risk of fractures. J. Bone Miner. Res. 16, 581-588 (2001).

38. Hubbard, R. et al. Use of inhaled corticosteroids and the risk of fracture*. Chest 130, 1082-1088 (2006).

39. Pujades-Rodrigues, M., Smith, C. J. P. \& Hubbard, R. B. Inhaled corticosteroids and the risk of fracture in chronic obstructive pulmonary disease. Q. J. Med. 100, 509-517 (2007).

40. Ye, Q., He, X. Q. \& D' Urzo, A. A review on the safety and efficacy of inhaled corticosteroids in the management of asthma. Pulm. Ther. 3, 1-18 (2017).

41. Curtis, H. J. \& Goldacre, B. OpenPrescribing: normalised data and software tool to research trends in English NHS primary care prescribing 1998-2016. BMJ Open 8, e19921 (2018)

\section{ACKNOWLEDGEMENTS}

The authors would like to thank Dr. Alex Walker for helping with understanding of OpenPrescribing data. The study was funded by a research award from the British Medical Association (BMA).

\section{AUTHOR CONTRIBUTIONS}

C.V.C. had full access to all the study data and takes full responsibility for the integrity of the data and the accuracy of the data analysis. Conception and design: C.V.C., D.E. S., T.M.M.; acquisition of data: C.V.C.; analysis of data: C.V.C.; interpretation of data: C.V. C., D.E.S., T.M.M.; drafting the article: C.V.C., D.E.S., T.M.M.; revision for important intellectual content and approval of the version to be published: C.V.C., D.E.S., T.M.M.

\section{COMPETING INTERESTS}

The authors declare no competing interests. 


\section{ADDITIONAL INFORMATION}

Supplementary information is available for this paper at https://doi.org/10.1038/ s41533-020-0162-6.

Correspondence and requests for materials should be addressed to C.V.C.

Reprints and permission information is available at http://www.nature.com/ reprints

Publisher's note Springer Nature remains neutral with regard to jurisdictional claims in published maps and institutional affiliations.
Open Access This article is licensed under a Creative Commons Attribution 4.0 International License, which permits use, sharing, adaptation, distribution and reproduction in any medium or format, as long as you give appropriate credit to the original author(s) and the source, provide a link to the Creative Commons license, and indicate if changes were made. The images or other third party material in this article are included in the article's Creative Commons license, unless indicated otherwise in a credit line to the material. If material is not included in the article's Creative Commons license and your intended use is not permitted by statutory regulation or exceeds the permitted use, you will need to obtain permission directly from the copyright holder. To view a copy of this license, visit http://creativecommons. org/licenses/by/4.0/.

(c) The Author(s) 2020 